\title{
COMMENT
}

\section{Comment on 'Analytical solution of Mori's equation with hyperbolic secant memory'}

\author{
M Howard Lee†, Jangil Kim $\dagger$, William P Cummings $\dagger$ and Raf Dekeyser \\ † Department of Physics and Astronomy, The University of Georgia, Athens, GA 30602-2451, \\ USA \\ $\ddagger$ Instituut voor Theoretische Fysica, Katholieke Universiteit Leuven, B-3001 Heverlee, \\ Belgium
}

Received 29 September 1994, in final form 10 January 1995

\begin{abstract}
The hyperbolic secant memory function recently proposed by Tankeshwar and Pathak has been analysed using the recurrence relations formulation. In particular, the relevant Hilbert space structure for this memory function has been deduced and compared with those of others obtained exactly from Hermitian many-body models. The realizability of the proposed memory function in conventional many-body models is further discussed.
\end{abstract}

\section{Introduction}

The memory function is a fundamental quantity needed to describe non-equilibrium behaviour in fluids. For a system with a realistic potential it has not been possible to obtain an exact closed-form memory function from first principles. One way to circumvent this difficulty is to seek functions with which to represent the memory function approximately. These functions must necessarily satisfy at least some essential properties of the memory function.

In an interesting article recently, Tankeshwar and Pathak [1] have proposed a hyperbolic secant as a memory function. This function satisfies several basic properties of a memory function. Hence, it is at once plausible but in addition, it has the advantage of being simple, flexible and convenient for dynamical analysis as these workers have demonstrated. Rather persuasive also are their results, showing good agreement with those of purely numerical origin.

Tankeshwar and Pathak contend that a hyperbolic secant memory is not without some physical basis at least macroscopically. It would nevertheless be very remarkable if a single function such as the hyperbolic secant could incorporate even approximately all the complexities of the memory in a fluid [2]. Hence, it would seem reasonable to examine this 'ersatz' memory function by microscopic theory to see what it implies both physically and analytically. There might possibly be some limitations not readily apparent.

Microscopically, the hyperbolic secant memory function has a few attractive features as well. It is time-reversal invariant, which is required in a Hermitian many-body system. At short times it appears oscillatory, but at long times it decays exponentially. It is an admissible function as we shall see but it does not allow the slow decay observed in other Hermitian many body models [3].

The main purpose of this comment is to show the microscopic conditions necessary for this memory function and to see whether they might be realizable. In recent years there 
have been several exact calculations of the relaxation function and the memory function for certain solvable models of quantum fluids and lattice spins (for reviews, see [4]; see also $[5,6])$. These results have indicated the existence of a variety of possible time evolutions and have provided an understanding of their microscopic origin.

These developments have been brought about in part by the recurrence relations approach to Mori's original memory function formalism [7]. Central to the recurrence relations approach is the realized Hilbert space of a dynamical variable (e.g. the velocity operator in the work of Tankeshwar and Pathak), spanned by a complete set of basis vectors. These basis vectors satisfy a recurrence relation and contain microscopic information in a precisely defined way. There are two structural properties which are essential to generating time evolution: the dimensionality and the hypersurface. The dimensionality of a realized space is usually not finite for a many-body model with infinite degrees of freedom. (It may on occasion not be infinite owing for example to localization.) The hypersurface is a measure of the norms of the basis vectors. The time evolution of a dynamical variable means the delineation of a trajectory on the surface of this Hilbert space, i.e. a particular structure and a particular time evolution are in effect equivalent. Given a particular time evolution such as hyperbolic secant, it is pertinent to ask what is the microscopic condition to which to ascribe.

\section{Recurrence relations formalism}

The recurrence relations formulation is briefly summarized here to analyse the hyperbolic secant memory function of Tankeshwar and Pathak. If $\boldsymbol{A}(t)$ is a dynamical variable at time $t$ for a model defined by a Hamiltonian $H$, the proper equation of motion is the generalized Langevin equation (GLE)

$$
\frac{\mathrm{d} A(t)}{\mathrm{d} t}+\int_{0}^{t} M\left(t-t^{\prime}\right) \boldsymbol{A}\left(t^{\prime}\right) \mathrm{d} t^{\prime}=F(t)
$$

where $M$ is the memory function and $F$ is the random force [8]. Tankeshwar and Pathak have started with a scalar version of (1). The formal solution of $A(t)$ according to the recurrence relations formalism is as follows [7]: $\boldsymbol{A}(t)$ is a vector in a $d$-dimensional Hilbert space $\mathcal{S}$, spanned by basis vectors $f_{0}, f_{1}, \ldots, f_{d-1}$ where $\left(f_{\nu}, f_{\nu^{\prime}}\right)=0$ if $v^{\prime} \neq v$. Hence,

$$
\boldsymbol{A}(t)=\sum_{\nu=0}^{d-1} a_{\nu}(t) f_{\nu}
$$

where $\left\{a_{\nu}\right\}$ is a family of real functions, e.g. $a_{0}(t)=\left(A(t), f_{0}\right) /\left(f_{0}, f_{0}\right) ; a_{\nu}$ is sometimes referred to as the relaxation function. The dimensionality $d$ is model dependent.

The distinguishing feature of this approach is that both $\left\{f_{\nu}\right\}$ and $\left\{a_{\nu}\right\}$ satisfy unique recurrence relations:

$$
\begin{aligned}
& f_{\nu+1}=\dot{f}_{\nu}+\Delta_{\nu} f_{\nu-1} \quad \nu \geqslant 0 \\
& \Delta_{\nu+1} a_{\nu+1}(t)=-\frac{\mathrm{d}}{\mathrm{d} t} a_{\nu}(t)+a_{\nu-1}(t) \quad v \geqslant 0
\end{aligned}
$$

where $\Delta_{\nu}=\left(f_{\nu}, f_{v}\right) /\left(f_{v-1}, f_{v-1}\right), \dot{f}_{\nu}=\mathrm{i}\left[H, f_{\nu}\right], f_{-1} \equiv 0$ and $a_{-1} \equiv 0$. By exercising the allowed freedom, e.g. $f_{0}=\boldsymbol{A}$, we can generate other $f_{\nu}$ and $\Delta_{\nu}$ from (3a). If $a_{0}$ were 
given, we could similarly generate other $a_{\nu}$ and $\Delta_{\nu}$ from ( $3 b$ ). However, it is not given and it must be determined from a known set of $\Delta_{v}$.

The random force $F(t)$ is a vector in a subspace $\mathcal{S}_{1}$, spanned by $f_{1}, f_{2}, \ldots, f_{d-1}$ :

$$
\boldsymbol{F}(t) \equiv f_{1}[t]=\sum_{\nu=1}^{d-1} b_{\nu}(t) f_{\nu}
$$

where $\left\{b_{v}\right\}$ is another family of real functions satisfying the same recurrence relation (3b) but in the subspace $\mathcal{S}_{1}$. The memory function follows from (4) at once:

$$
M(t) \equiv M_{1}(t)=\left(f_{1}[t], f_{1}\right) /\left(f_{0}, f_{0}\right)=\Delta_{1} b_{1}(t) .
$$

The time evolution of $F(t) \equiv f_{1}[t]$ is itself also governed by the GLE (1). If $A(t)$ is replaced by $f_{1}[t]$ therein, it introduces a new random force $f_{2}[t]$ and a new memory function $M_{2}(t)$. Then $f_{2}[t]$ is a vector in a subspace $\mathcal{S}_{2}$, spanned by $f_{2}, f_{3}, \ldots, f_{d-1}$ :

$$
f_{2}[t]=\sum_{\nu=2}^{d-1} c_{\nu}(t) f_{v}
$$

where $\left\{c_{v}\right\}$ also satisfy the same recurrence relation $(3 b)$ in the subspace $\mathcal{S}_{2}$. The memory function is

$$
M_{2}(t)=\left(f_{2}[t], f_{2}\right) /\left(f_{1}, f_{1}\right)=\Delta_{2} c_{2}(t)
$$

Evidently we can continue this process until the smallest subspace is reached if $d$ is finite or indefinitely if $d$ is not finite. There is an invariant relationship between these spaces. Thus the random force and the memory function in one space, for example, can be formally viewed as the dynamical variable and the relaxation function in its subspace. The invariant relationship is most clearly indicated by the Laplace transforms. Let $\hat{a}_{\nu}(z)=L a_{\nu}(t)$, where $L$ is the Laplace transform operator. Then, by $(3 b)$,

$$
z \hat{a}_{0}(z)+\Delta_{1} \hat{a}_{1}(z)=1 .
$$

Hence,

$$
\hat{a}_{0}(z)=\left[z+\Delta_{1} \hat{b}_{1}(z)\right]^{-1}
$$

and

$$
\hat{a}_{1}(z)=\hat{b}_{1}(z) \hat{a}_{0}(z)
$$

Similarly,

$$
\hat{b}_{1}(z)=\left[z+\Delta_{2} \hat{c}_{2}(z)\right]^{-1}
$$

etc. These and similar relationships imply that

$$
\hat{a}_{0}(z)=\frac{1}{z+\frac{\Delta_{1}}{z+\frac{\Delta_{2}}{z+\cdots+\frac{\Delta_{d-1}}{z}}}}
$$

i.e. a continued fraction.

If $\left\{\Delta_{\nu}\right\}$ is calculated from (3a), $\hat{a}_{0}(z)$ may be determined from (9). Then $a_{0}(t)$ can be found from $\hat{a}_{0}(z)$ by an inverse transform. The recurrence relation $(3 b)$ is sufficient to determine $\left\{a_{v}(t)\right\}$. Also $\hat{b}_{1}(z)$ can be found from $(8 c)$ and $b_{1}(t)$ by a convolution and so on. If the time evolution is determined in this manner, we shall term it canonical. A canonically obtained relaxation function is perforce an admissible function [7]. 


\section{Analysis of the memory function of Tankeshwar and Pathak}

Given the proposition $M(t)=\operatorname{sech} t$, we can use $(3 b)$ to construct the Hilbert space structure immediately. Since the memory function in the space $\mathcal{S} \equiv \mathcal{S}_{0}$ is also the relaxation function in the subspace $\mathcal{S}_{\mathrm{I}}$ by the invariant relationship, the analysis can be carried out wholly in this subspace regarding sech $t$ as the relaxation function therein. However, it is simpler to regard it as the relaxation function in the space $\mathcal{S}_{0}$ and thereby to avoid relabelling. This translation does not affect our intended analysis.

Let $a_{0}(t)=$ sech $t$. Then, by repeated applications of $(3 b)$, we obtain $a_{\nu}(t)=$ $\left(\tanh ^{v} t \operatorname{sech} t\right) / \nu$ ! and $\Delta_{\nu}=v^{2}, \nu \geqslant 1, d=\infty$. It is important to recognize that sech $t$ is an admissible function unlike $\exp (-t)$ for example (see appendix). This particular infinitedimensional hypersurface can also be obtained from a continued-fraction representation (9) due to Stieltjes [9]:

$$
\begin{aligned}
& \hat{a}_{0}(z)=\int_{0}^{\infty} \exp (-z t) \operatorname{sech} t \mathrm{~d} t \quad \operatorname{Re} z>0 \\
& \hat{a}_{0}(z)=\frac{1}{z+\frac{1^{2}}{z+\frac{2^{2}}{z+\frac{3^{2}}{z+\cdots}}}}
\end{aligned}
$$

Before analysing the above result, we shall list for comparison three representative results of infinite dimensions obtained canonically.

(a) $\Delta_{\nu}=1, v \geqslant 1 ; d=\infty$. This structure occurs in the 2D electron gas and in the classical harmonic oscillator chain $[10,11,17]$ :

$$
a_{0}(t)=2 J_{1}(t) / t \quad \hat{a}_{0}(z)=\left(\sqrt{z^{2}+4}-z\right) / 2 .
$$

(b) $\Delta_{v}=v, v \geqslant 1 ; d=\infty$. This structure occurs in the spin van der Waals model [12] and in the spin $X Y$ chain [13]:

$$
a_{0}(t)=\exp \left(-\frac{t^{2}}{2}\right) \quad \hat{a}_{0}(z)=\frac{z}{\sqrt{\pi}} \int_{0}^{\infty} \frac{\exp (-u) u^{-1 / 2} \mathrm{~d} u}{2 u+z^{2}} \quad \operatorname{Re} z \neq 0 .
$$
[14]:

(c) $\Delta_{\nu}=v^{2} /\left(4 v^{2}-1\right), v \geqslant 1 ; d=\infty$. This structure occurs in the 3D electron gas

$$
a_{0}(t)=j_{0}(t) \quad \hat{a}_{0}(z)=\tan ^{-1} z^{-1} .
$$

Observe that all the singularities of $\tilde{a}_{0}(z)$, whether isolated poles or branch points, lie on the imaginary axis of $z$. If these structures are perturbed (e.g. by adding an impurity to a harmonic oscillator chain (see (a))) [11], additional isolated poles occur which are also found on the imaginary axis, corresponding to resonant frequencies. (Recall that $z=i \omega+0$, where $\omega$ is the frequency.) In all exact results obtained canonically [4], there have been no exceptions.

Now let us examine the singularities of $\hat{a}_{0}(z)$ when $\Delta_{\nu}=\nu^{2}, \nu \geqslant 1$, corresponding to $a_{0}(t)=\operatorname{sech} t$. It is convenient to write $[7,15]$

$$
\hat{a}_{0}(z)=Q(z) / P(z)=\lim _{N \rightarrow \infty}\left\{\operatorname{det}\left[\mathbf{Q}_{N}(z)\right] / \operatorname{det}\left[\mathbf{P}_{N}(z)\right]\right\}
$$


and to look for the zeros of $P(z)$, where $P_{N}(z)$ is a tridiagonal matrix:

$$
\mathbf{P}_{N}=\left[\begin{array}{cccccc}
z & -1 \mathrm{i} & & & & \\
-1 \mathrm{i} & z & -2 \mathrm{i} & & & \\
& -2 \mathrm{i} & z & -3 \mathrm{i} & & \\
& & -3 \mathrm{i} & \ddots & & \\
& & & & \ddots & -N \mathrm{i} \\
& & & & -N \mathrm{i} & z
\end{array}\right] \text {. }
$$

$\mathbf{Q}_{N}(z)$ is also a similar tridiagonal matrix but not needed here since we may safely assume that $Q(z)$ is regular.

The zeros of $P(z)$ are the eigenvalues of the above matrix as $N \rightarrow \infty$. They may be obtained by a method due to Lee et al [16] based on a generating function. The eigenvalues are $z=-1,-3,-5, \ldots$. We can easily verify the above result as follows:

$\hat{a}_{0}(z)=\int_{0}^{\infty} \exp (-z t) \operatorname{sech} t \mathrm{~d} t=\frac{1}{2}\left[\psi\left(\frac{1}{4} z+\frac{3}{4}\right)-\psi\left(\frac{1}{4} z+\frac{1}{4}\right)\right] \quad \operatorname{Re} z>-1$

where $\psi$ is the logarithmic derivative of the $\Gamma$ function. $\psi(z)$ is meromorphic with simple poles at $z=0,-1,-2, \ldots$. Also $\operatorname{Re} \hat{a}_{0}(z=i \omega+0)=(\pi / 2) \operatorname{sech}\left(\frac{1}{2} \pi \omega\right)$. From (13), we can obtain the normalized dynamic structure factor $\tilde{S}(\omega)$, where $\omega$ is the frequency:

$\tilde{S}(\omega)=\frac{\pi^{-1}}{1-\exp (-\beta \omega)} \operatorname{Im}\left[z \hat{\alpha}_{0}(z)\right]_{z=\mathrm{i} \omega+0}= \begin{cases}\frac{1}{2} \omega \operatorname{sech}\left(\frac{1}{2} \pi \omega\right) & \text { (quantum) } \\ (1 / 2 \beta) \operatorname{sech}\left(\frac{1}{2} \pi \omega\right) & \text { (classical) }\end{cases}$

where $h=1$ and $\beta$ is the inverse temperature.

The properties of the subspace $\mathcal{S}_{1}$ may be determined from $(8 b)$ and $(9)$ as follows:

$$
\hat{b}_{1}(z)=\frac{1}{z+\frac{2^{2}}{z+\cdots}}=-z+2\left[\psi\left(\frac{1}{4} z+\frac{3}{4}\right)-\psi\left(\frac{1}{4} z+\frac{1}{4}\right)\right]^{-1} .
$$

Note that $\hat{b}_{\mathrm{I}}(z=0)=2 / \pi$. Also,

$$
b_{1}(t \rightarrow 0)=1-2 t^{2}+\left(\frac{13}{6}\right) t^{4}-\cdots
$$

By the convolution theorem (see $(8 c)$ ),

$$
b_{1}(t \rightarrow \infty)=(2 / \pi) \tanh t \operatorname{sech} t \simeq(1 / \pi) \exp (-t)
$$

We can determine $c_{2}$ and its family similarly. In this manner, we can completely characterize all the subspaces $\mathcal{S}_{1}, \mathcal{S}_{2}, \ldots$ from knowledge of the space $\mathcal{S}_{0}$.

The above picture for the structure $\Delta_{\nu}=\nu^{2}, v \geqslant 1$, is generalizable slightly by taking $a_{0}(t)=\operatorname{sech}^{k} t, k=1,2, \ldots$. Then, from $(3 b)$, we obtain $a_{\nu}(t)=\left(\tanh ^{v} t \operatorname{sech}^{k} t\right) / \nu !$, $\Delta_{\nu}=v(v+k-1), v \geqslant 1$, and $d=\infty$. The eigenvalues of $\mathbf{P}_{N}(z)$ whose off-diagonal elements are $-\mathrm{i} \sqrt{1 k},-\mathrm{i} \sqrt{2(k+1)},-\mathrm{i} \sqrt{3(k+2)}, \ldots$ are $z=-2 m-k, m=0,1,2, \ldots$, if $N \rightarrow \infty$. 


\section{Concluding remarks}

The singularities of $\hat{a}_{0}(z)$ when $\Delta_{\nu}=v^{2}, v \geqslant 1$, are real (i.e. imaginary frequencies). They are isolated poles, all lying on the negative real axis of $z$. This behaviour is unaltered if $\Delta_{v}=v(v+k-1), v \geqslant 1$, for $k=1,2, \ldots$. This is in contrast with the behaviour of the physical singularities in the three examples $(a)-(c)$ of section 3 as well as in all other known solutions obtained canonically [4]. For all these the singularities lie on the imaginary axis of $z$, corresponding to real frequencies. Apparently there is a fundamental difference between the sech $t$ relaxation function and the canonically obtained relaxation functions. Since all of them including the hyperbolic secant (see appendix) are admissible functions, the difference can only be bound to the question of realizability.

Let us therefore look at this question more closely. The class of these canonically obtained $a_{v}(t)$, e.g. $a_{v}(t)=\left[t^{\nu} \exp \left(-\frac{1}{2} t^{2}\right)\right] / \nu$ !, which represent the structure of $\Delta_{v}=v$, $v \geqslant 1$, are all entire functions of $t$. However, the functions $a_{\nu}(t)=\left(\tanh ^{\nu} t \operatorname{sech} t\right) / \nu$ !, which represent the structure of $\Delta_{v}=v^{2}, v \geqslant 1$, are not. This is further illuminated if viewed from a growth process where we define an exponent $x$ by $\Delta_{v} \sim \nu^{x}, v \rightarrow \infty$. It has been argued that, if $x \geqslant 2$, there is infinite growth order and the relaxation function is not entire $[5,18]$. Not coincidentally, $x=2$ for $a_{0}(t)=\operatorname{sech} t$, but $x<2$ for the canonically obtained $a_{\nu}(t)$ (see $(a)-(c)$ of section 3 ). In this growth sense the hyperbolic secant is at an onset of infinite growth, whereas the others are in the domain of finite growth.

Can the Hilbert space structure of $\Delta_{v}=v^{2}, \nu \geqslant 1$, be realized in many-body models at all? Given the analytical constraint for this structure, it does not seem likely if models have smooth potentials, but it is possible if models have non-smooth potentials such as a hard-sphere potential. That exactly the same analytical property should also occur in a quantum hard-sphere model is highly suggestive [16]. In these models this Hilbert space structure may very well be realized.

Finally let us now look at a hyperbolic secant memory more physically. We observed at the outset that a hyperbolic secant memory has no slow decay. It is not dissimilar to what is seen in stochastic models, where memories tend to become lost after short times. Indeed we expect to find this kind of dynamical behaviour in models with contact potentials, for which a hyperbolic secant memory should give a good account. Slow decay, however, does exist in more realistic models for fluids and has been observed in fluids. To be viable for fluids, a hyperbolic secant memory would need to be supplemented with slowly vanishing components. Otherwise their dynamical behaviour especially in low-frequency regimes would be poorly accounted for by a hyperbolic secant memory alone.

\section{Acknowledgments}

One of us (MHL) wishes to thank Professor K N Pathak for correspondence regarding the proposed memory function. This work has been supported in part by NSF and NATO (CRG 921268).

\section{Appendix. Admissibility of sech $t$}

In the recurrence relations formalism [7] a function is admissible as the relaxation function (or the memory function) if it satisfies the Bessel equality:

$$
\sum_{v=0}^{\infty} a_{v}^{2}(t) \prod_{k=1}^{\nu} \Delta_{k}=1 .
$$


This condition can be used to show that a purely exponential function is inadmissible [3].

One can prove that sech $t$ is admissible by satisfying (A1). If $a_{0}(t)=\operatorname{sech} t$,

$$
\Delta_{\nu}=\nu^{2}
$$

and

$$
a_{\nu}(t)=\frac{\tanh ^{\nu} t \operatorname{sech} t}{\nu !}
$$

Let us write for simplicity $T=\tanh t$ and $C=\cosh t=(\operatorname{sech} t)^{-1}$. Then, the left-hand side of (A1) is

$$
\sum_{\nu=0}^{\infty} \frac{1}{(v !)^{2}} \frac{T^{2 v}(v !)^{2}}{C^{2}}=\frac{1}{C^{2}} \frac{1}{\left(1-T^{2}\right)}=1 \quad \text { QED }
$$

\section{References}

[1] Tankeshwar K and Pathak K N 1994 J. Phys.: Condens, Matter 6591

[2] Tsekov R and Radoev B 1992 J. Phys.: Condens. Matter 4 L303

Lee M H 1992 J. Phys.: Condens. Matter 410487

[3] Lee M H 1983 Phys. Rev. Lett. 511227

[4] Pires A S T 1988 Helv. Phys. Acta 61988

Lee M H, Hong J and Florencio J 1987 Phys. Scr. T 19498

Lee M H, Kim J, Cummings W P and Dekeyser R $1995 \mathrm{~J}$. Mol. Struct. at press

[5] Viswanath V S and Müller G 1994 The Recursion Method (Berlin: Springer)

[6] Grigolini P 1993 Quantum Mechanical Irreversibility and Measurement (Singapore: World Scientific)

[7] Lee M H 1982 Phys. Rev. B 26 2547; 1982 Phys. Rev. Lett. 491072

[8] Mori H 1965 Prog. Theor. Phys. 33423

Zwanzig R 1965 Ann. Rev. Chem. Phys. 1667

[9] Wall H S 1948 Analytical Theory of Continued Fractions (New York: Chelsea) pp 206, 372

[10] Lee M H and Hong J 1982 Phys. Rev. Lett. 48 634; 1985 Phys. Rev. B 327734

[11] Lee M H, Florencio J and Hong J 1989 J. Phys. A: Math. Gen. 22 L331

[12] Lee M H, Kim I M and Dekeyser R 1984 Phys. Rev. Lett. 521579

[13] Florencio J and Lee M H 1987 Phys. Rev. B 351835

[14] Lee M H and Hong J 1984 Phys. Rev. B 306756

[15] Stone M H 1974 Linear Transformation in Abstract Hilbert Space (Providence, RI: American Mathematical Society)

[16] Lee T D, Huang K and Yang C N 1957 Phys. Rev. 1061136 Appendix 1

[17] Sen S, Mahanti S D and Cai Z X 1991 Phys. Rev. B 4310990

[18] Böhm M, Leschke H, Henneke M, Viswanath V S, Stoize J and Müller G 1994 Phys. Rev, B 49417 